\title{
Mathematical Model of Blood Flow in Small Blood Vessel in the Presence of Magnetic Field
}

\author{
Rekha Bali, Usha Awasthi \\ Department of Mathematics, Harcourt Butler Technological Institute, Navabganj, India \\ E-mail:dr.rekhabali@rediffmail.com, usha_hbti@rediffmail.com \\ Received December 14, 2010; revised January 3, 2011; accepted January 8, 2011
}

\begin{abstract}
A mathematical model for blood flow in the small blood vessel in the presence of magnetic field is presented in this paper. We have modeled the two phase model for the blood flow consists of a central core of suspended erythrocytes and cell-free layer surrounding the core. The system of differential equations has been solved analytically. We have obtained the result for velocity, flow rate and effective viscosity in presence of peripheral layer and magnetic field .All the result has been obtained and discussed through graphs.
\end{abstract}

Keywords: Blood, Plasma, Magnetic Field, Effective Viscosity, Peripheral Layer

\section{Introduction}

Blood shows anomalous viscous properties. The anomalous behavior of blood is principally due to the suspension of particles in plasma. The plasma solution in the blood obeys the linear Newtonian model for viscosity [1]. However, blood as a whole is often considered as nonNewtonian fluid, particularly when the characteristic dimension of the flow is close to the cell dimension. The experimental observations and theoretical analysis of blood flow are very useful for the diagnosis of a number of cardiovascular diseases and development of pathological patterns in animal or human physiology [2]. The flow of blood through small diameter tubes is of physiological and clinical importance. Due to its complexity and anomalous behaviour, it is very difficult to analyze it. The two types of anomaly are due to low shear and high shear effects [3]. When blood flows through larger diameter arteries at high shear rates, it behaves like a Newtonian fluid. The apparent viscosity of blood decreases with decreasing blood vessel diameter, when measurements are made in capillaries of diameter less than $300 \mu \mathrm{m}$ [4].

Pries et al. [5] studied the effect of the tube diameter and the hematocrit ratio on the blood viscosity and found that for tube diameters greater than $1 \mathrm{~mm}$, the blood viscosity is independent of the diameter while for tube diameter less than $1 \mathrm{~mm}$, the blood viscosity is strongly dependent on the tube diameter. They also reported the viscosity increases non-linearly with the hematocrit. Bug- liarello and Sevilla [6], Cokelet [7] and [8-10] have reported that for blood flowing through narrow blood vessels, there is a peripheral layer of plasma and a core region of suspension of all the erythrocytes.

Also the red blood cell is major bio-magnetic substance and the blood flow may be influenced by the magnetic field [11]. The effect of magnetic field on blood flow has been analyzed theoretically by treating blood as an electrically conductive fluid [12]. Assuming blood as a magnetic fluid, it may be possible to control blood pressure and its flow behavior by using an appropriate magnetic field. Hence such studies have potential for therapeutic use in the diseases of heart, blood and blood vessels.

Most of the model [13-17] on blood flow deal with one phase model. However, in view of the fact that blood is a suspension, a two-phase model appears to be more appropriate. Wagh and Wagh [18] have used [19] a model of dusty gas to study the effect of the magnetic nature of red blood cells of the flow of blood. The reason is, blood is a liquid suspension having mass and volume concentrations roughly the same, however, for dusty gas the mass and bulk concentrations are quite different [20], Nayfeh's [21] two phase model seems to be more suitable for blood flow.

In view of the above mentioned fact, we have considered the two phase model consisting of central core of suspended erythrocyte and cell free layer surrounding the core in the presence of magnetic field. 


\section{Mathematical Analysis}

We have considered a two layer model (Figure 1) for the blood flow within cylindrical vessel of radius $\mathrm{R}$ consisting of central core radius $R_{1}$, which contains an erythrocyte suspension of uniform hematocrit and a cell free layer outside the core containing plasma. We have taken some assumptions for formulating the mathematical model.

Blood is considered as viscous, incompressible and electrically conducting fluid. Fluid flow is steady and laminar. Magnetic field is constant in transverse direction.

\subsection{Governing Equations}

Introducing the assumptions mentioned above the governing equation for the fluid flow are given as.

$$
\begin{gathered}
\frac{\partial P^{\prime}}{\partial z^{\prime}}=\frac{\mu_{0}}{r^{\prime}} \frac{\partial}{\partial r^{\prime}}\left(r^{\prime} \frac{\partial u_{0}^{\prime}}{\partial r^{\prime}}\right), R_{1}<r^{\prime}<R_{0} \\
0=(1-\phi)\left[-\frac{\partial P^{\prime}}{\partial z^{\prime}}+\mu_{s}(\phi)\left(\frac{\partial^{2} u_{f}^{\prime}}{\partial r^{\prime 2}}+\frac{1}{r^{\prime}} \frac{\partial u_{f}^{\prime}}{\partial r^{\prime}}\right)\right] \\
+\phi F_{d}\left(u_{p}^{\prime}-u_{f}^{\prime}\right) \\
0=\phi\left[-\frac{\partial P^{\prime}}{\partial z^{\prime}}-F_{d}\left(u_{p}^{\prime}-u_{f}^{\prime}\right)+k_{0} M_{p} \frac{d H^{\prime}}{d z^{\prime}}\right]
\end{gathered}
$$

Where $r^{\prime}, z^{\prime}$ are radial and axial co-ordinate, $u_{f}$ and $u_{p}$ are the velocities of the fluid (plasma) and particles (red cell), $\phi$ is the volume fraction of the red cells, $\mu_{s}(\phi)$ is the suspension viscosity, $F_{d}$ is the drag coefficient of interaction for the force exerted by one phase on the other, $k_{0}$ is the magnetic permeability, $M_{p}$ is the magnetization of red cells and $\frac{d H^{\prime}}{d z^{\prime}}$ is the magnetic field gradient. The expression of the drag coefficient is given by:

$F_{d}=\frac{9}{2} \frac{\mu_{f}}{R^{2}} \lambda(\varphi)$, where $\lambda(\varphi)=\frac{4+3\left(8 \varphi-3 \varphi^{2}\right)^{\frac{1}{2}}+3 \varphi}{(2-3 \varphi)^{2}}$

Where $\mu_{f}$ is the constant fluid viscosity.

The viscosity of the suspension is given by an empirical relation.

$$
\begin{array}{cr}
\mu_{s}=\frac{\mu_{f}}{(1-\beta \varphi)}, \text { when } & u_{0}=\frac{R_{e}}{4} \varepsilon \frac{\partial P}{\partial z}\left(R_{1}^{2}-1\right) \\
u_{f}=\frac{R_{e}}{4} \frac{\partial P}{\partial z}\left[\frac{1}{(1-\phi) \mu_{s}}\left\{e \phi R^{2}+(1-\phi) \varepsilon\right\}\left(r^{2}-R_{1}^{2}\right)+\varepsilon\left(R_{1}^{2}-1\right)\right]-\frac{\phi R_{e} R^{2} e M_{p} k_{0}}{4(1-\phi) \mu_{s}} \cdot \frac{d H}{d z}\left(r^{2}-R_{1}^{2}\right)
\end{array}
$$




$$
u_{p}=\frac{R_{e}}{4} \frac{\partial P}{\partial z}\left[\frac{1}{(1-\phi) \mu_{s}}\left\{e \phi R^{2}+(1-\phi) \varepsilon\right\}\left(r^{2}-R_{1}^{2}\right)+\varepsilon\left(R_{1}^{2}-1\right)-\frac{4 e}{F_{d}}\right]+R_{e} M_{p} k_{0} \frac{d H}{d z}\left[\frac{e}{F_{d}}-\frac{\phi R^{2} e}{4(1-\phi) \mu_{s}}\left(r^{2}-R_{1}^{2}\right)\right]
$$

The flow flux (volumetric flow rate) is now calculated as

$$
\begin{gathered}
Q=Q_{0}+Q_{f}+Q_{p} \quad \text { (9) } \quad \text { pression for flow rate is obtained as: } \\
Q=\frac{\pi R^{4}}{8 \mu_{s}} \frac{\partial P}{\partial z} U_{0} R_{e} \mu_{s} \varepsilon\left(1-R_{1}^{2}\right)\left(R^{2}+R_{1}^{2} R^{2}-2\right)+\frac{2 R_{1}^{4} U_{0} R_{e}}{(1-\phi)} \cdot\left\{\left(e \phi R^{2}+(1-\phi) \varepsilon\right)\left(R^{2}-2\right)+\frac{2 \varepsilon}{R_{1}^{2}}\left(R_{1}^{2}-1\right)\right\} \\
+\frac{4 \phi U_{0} R_{e} \mu_{s} e}{F_{d}}+\frac{8 \phi R_{1}^{4} U_{0} R_{e} M_{p} k_{0} e \mu_{s}}{\left(\frac{\partial P}{\partial z}\right)} \cdot \frac{d H}{d Z}\left\{\frac{1}{F_{d} R_{1}^{2}}-\left(R^{4}-2 R^{2}\right)\left(1+\frac{4 \phi}{(1-\phi) \mu_{s}}\right)\right\}
\end{gathered}
$$

Using Equations (6)-(8) in the Equation (9) the ex-
Using the fact that total flux is equal to the sum of the fluxes across the two regions (peripheral and core) determines the relation.

$$
R_{1}=\alpha R
$$

Using the relation (10) and (11), the expression for the effective (apparent) viscosity is given by:

$$
\begin{aligned}
\mu_{e}= & \mu_{s}\left[U_{0} R_{e} \mu_{s} \varepsilon\left(1-\alpha^{2} R^{2}\right)\left(R^{2}+R^{4} \alpha-2\right)+\frac{2 \alpha^{4} R^{4} U_{0} R_{e}}{(1-\phi)}\left\{\begin{array}{c}
\left(e R^{2} \phi+(1-\phi) \varepsilon\right)\left(R^{2}-2\right) \\
2 \varepsilon\left(\alpha^{2} R^{2}-1\right) \\
\alpha^{2} R^{2}
\end{array}\right\}\right. \\
& \left.-\frac{4 \phi U_{0} R_{e} \mu_{s} e}{F_{d}}+\frac{8 \phi \alpha^{4} R^{4} U_{0} R_{e} M_{p} k_{0} e \mu_{s}}{\left(\frac{\partial P}{\partial z}\right)} \cdot \frac{d H}{d z}\left\{\frac{1}{F_{d} \alpha^{2} R^{2}}-\left(R^{4}-2 R^{2}\right)\left(1+\frac{4 \phi}{(1-\phi) \mu_{s}}\right)\right\}\right]^{-1}
\end{aligned}
$$

\section{Results and Discussions}

To have a quantitave estimate of the various parameters involved, particularly the hematocrit $(\phi)$ and magnetic field gradient $\left(H_{Z}=\frac{\partial H}{\partial z}\right)$, some of the results is displayed graphically in Figures 2-8.

The variation of effective viscosity $\left(\mu_{e}\right)$ for different values of magnetic field gradient $\left(H_{z}\right)$ is shown in Figure 2 and for different values of hematocrit $(\phi)$ is shown in Figure 3. The effective viscosity increases with increasing value of hematocrit. Also, the effective viscosity increases with the increase of the magnetic field gradient. The major mechanism of the influence of a static magnetic field on blood flow viscosity is based on the interaction between the induced magnetic moment on the RBC and the external static magnetic field. This property in a static magnetic field increases the friction of the flowing blood, because the anisotropic orientation of the RBC in the static magnetic field distribus the rolling of the cell in flowing blood, and so the blood viscosity increases [22].

Figure 4 shows the variation of flow rate $(Q)$ with hematocrit of blood $(\phi)$ for different values of magnetic field gradient $\left(H_{z}\right)$. It is clear from the figure that the flow rate decreases slowly with the increase of the

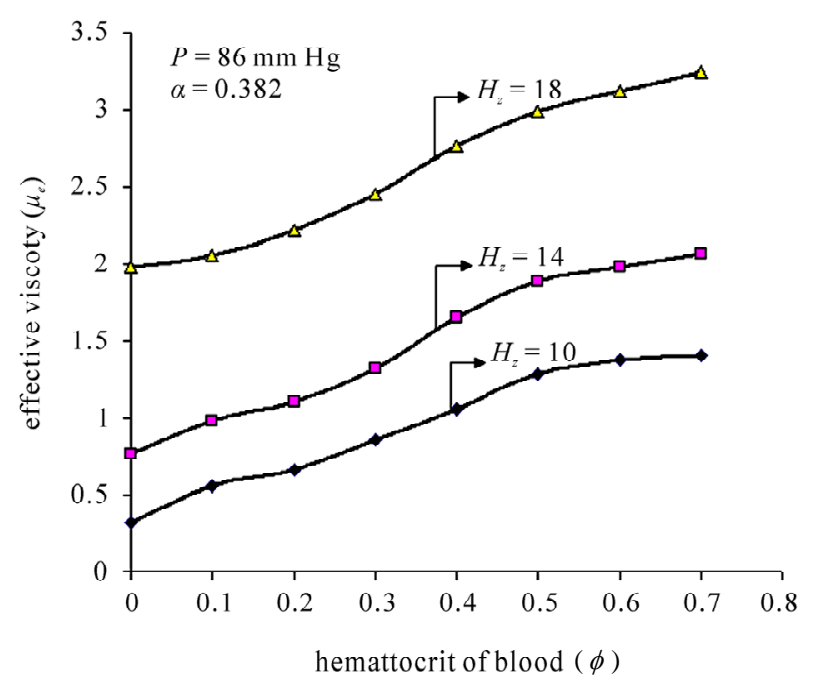

Figure 2. Variation of effective viscosity $\left(\mu_{e}\right)$ with hematocrit of blood $(\phi)$ for different values of magnetic field gradient $H_{z}=(d H / d z)$. 


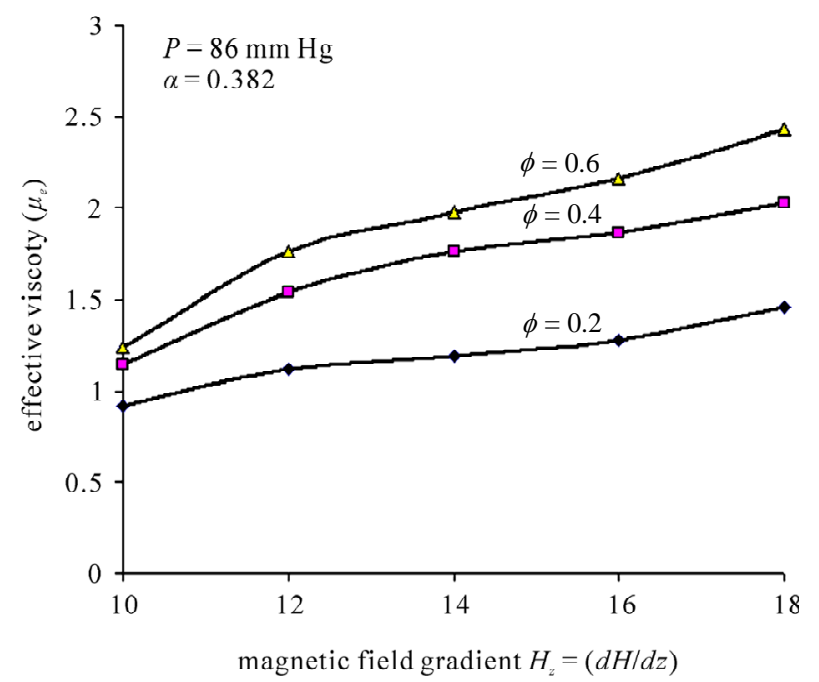

Figure 3. Variation of effective viscosity $\left(\mu_{e}\right)$ with magnetic field gradient $H_{z}=(d H / d z)$ for different values hematocrit $(\phi)$.

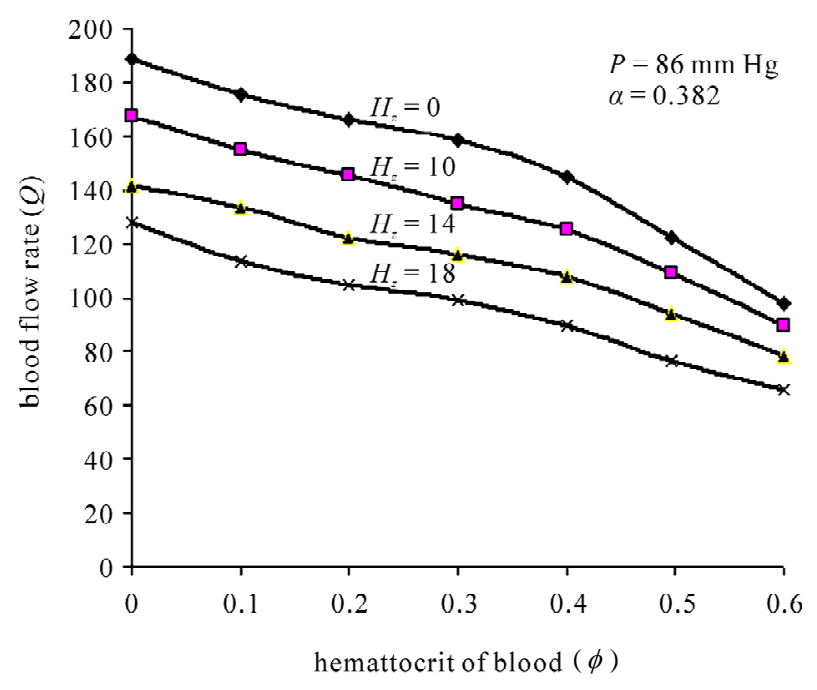

Figure 4. Variation of blood flow rate $(Q)$ with hematocrit of blood $(\phi)$ for different values of magnetic field gradient $H_{\mathrm{z}}=(\mathrm{dH} / \mathrm{dz})$.

hematocrit and decrease with increasing values of the magnetic field gradient. It may be observed that the flow rate is significantly influenced by the magnetic field gradient, the magnetic nature of the fluid and hematocrit of blood $(\phi)$.

The variation of axial velocities profiles $u_{f}$ and $u_{p}$ for both phase (plasma and erythrocyte) with radial axis (r) for different values of hematocrit $(\phi)$ are plotted in Figures 5 and 7. It has been observed that the $u_{f}$ and $u_{p}$ decrease with increase of the hematocrit $(\phi)$ for constant magnetic field gradient $\left(H_{z}\right)$ and other parameter are keep constant. The effect of hematocrit of blood $(\phi)$ on the velocity is relatively small near the wall. It may be due to the red cell's tendency to accumulate near the tube axis.

Figures 6 and 8, shows the variation of axial velocites profiles $u_{f}$ and $u_{p}$ for both phase (plasma and erythrocyte) with radial axis (r) for different values of magnetic field gradient $\left(H_{z}\right)$. It is clear from the figure that $u_{f}$ and $u_{p}$ decrease with increase of the magnetic field gradient. Thus, it is of importance to note that though the suspending fluid is non magnetic the magnetic field gradient influence its velocity.

All these results of the present study have been compared with already existing results obtained in the theoretical study of [2], [13] and [21].

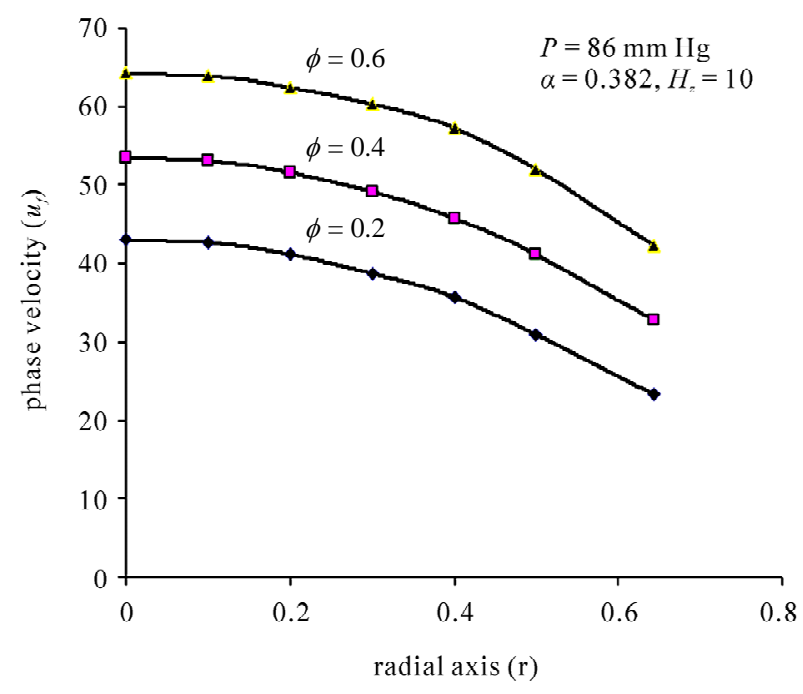

Figure 5. Variation of phase velocity $\left(u_{f}\right)$ with radial axis for different values of hematocrit of blood $(\phi)$.

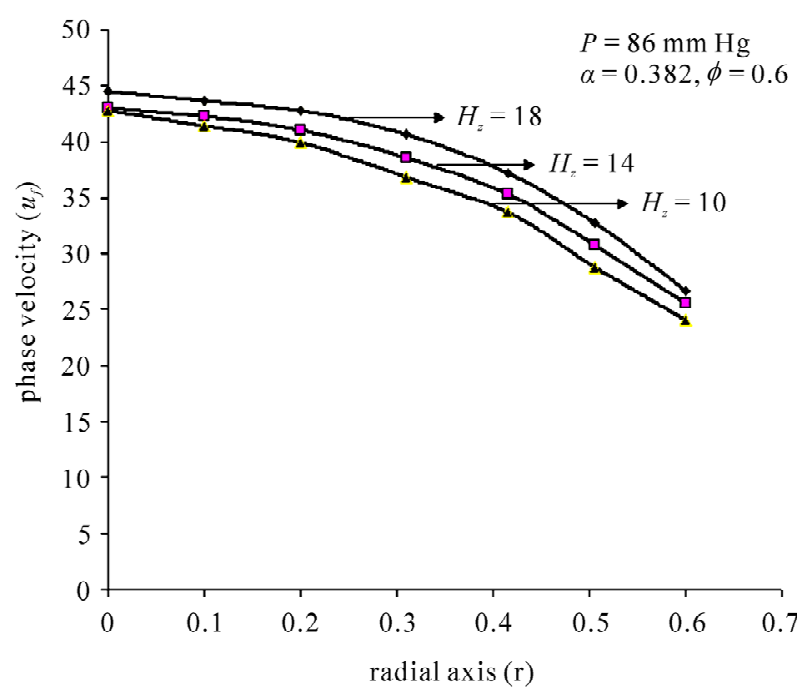

Figure 6. Variation of phase velocity $\left(u_{f}\right)$ with radial axis for different values of magnetic field gradient $H_{z}=(\mathrm{dH} / \mathrm{dz})$. 


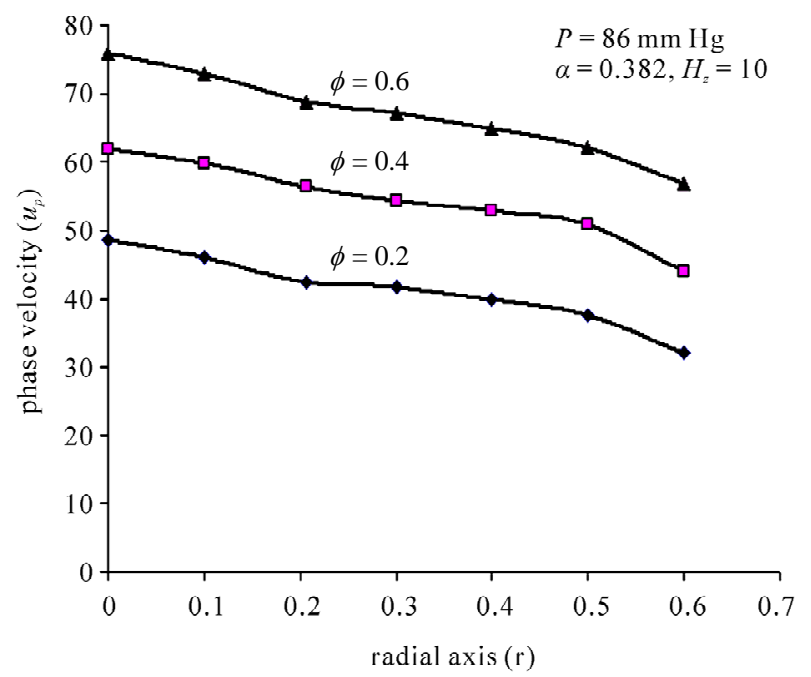

Figure 7. Variation of pluge velocity $\left(u_{p}\right)$ with radial axis for different values of hematocrit of blood $(\phi)$.

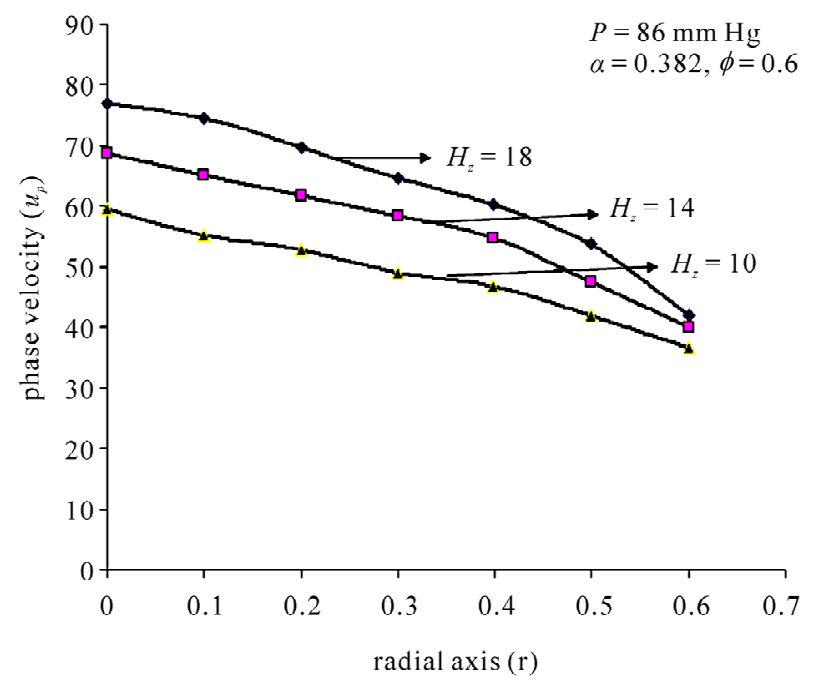

Figure 8. Variation of pluge velocity $\left(u_{p}\right)$ with radial axis for different values of magnetic field gradient $H_{z}=(\mathrm{dH} / \mathrm{dz})$.

\section{Conclusions}

This study brings out many interesting fluid mechanical phenomena due to the magnetic field and presence of the peripheral layer. Blood has been modeled as two-fluid model with the core region of suspension of all the erythrocytes and the plasma in the peripheral region as a Newtonian fluid. It is noted that the velocity and flow rate decreases, while the effective viscosity increases with magnetic field and hematocrit .

It is clear from the above discussion that magnetic field affects largely on the axial flow velocities of blood and effective viscosity. So, by taking appropriate values of magnetic field we may regulate the axial velocities and effective viscosity.

\section{References}

[1] G. R. Cokelet, Y. C. Fung, N. Perrone and M. Anliker, "Biomechanics-Its Foundations and Objectives," Prentice-Hall, Englewood Cliffs, 1972.

[2] V. P. Srivastava and R.Srivastava, "Particulate Suspension Blood Flow Through a Narrow Catheterized Artery," Computers \& Mathematics with Applications, Vol. 58, No. 2, 2009, pp. 227-238. doi:10.1016/j.camwa.2009.01.041

[3] S. Chien, S. Usami and R. Skalak, "Blood Flow in Small Tubes,” E. M. Renkins and C. C. Michel, Eds., American Physiological Society Handbook of Physiology, Section 2, The Cardiovascular System, 4, Bethesda MD, American Physiological Society, 1984, pp. 217-249.

[4] R. Fahraeus and R. Lindqvist, "The Viscosity of the Blood in Narrow Capillary Tubes," American Journal Physiology, Vol. 96, 1931, pp. 562-568.

[5] A. R. Pries, D. Neuhaus and P. Gaehtegnes, "Blood Viscosity in Tube Flow: Dependence on Diameter and Hematocrit,” American Journal of Physiology, Vol. 263, 1992, pp. 1170-1778.

[6] G. Bugliarello and J. Sevilla, "Velocity Distribution and Other Characteristics of Steady and Pulsatiles Blood Flow in Fine Glasss,” Biorheology, Vol. 7, No. 2, 1970, pp. 85-107.

[7] G. R. Cokelet, “The Rheology of Human Blood,” Y. C. Fung (Eds.), Biomechanics, Prentice-Hall, Englewood Cliffs, 1972.

[8] M. Sharan and A. S. Popel, “A Two-Phase Model for Flow of Blood in Narrow Tubes with Increased Effective Viscosity Near the Wall,” Biorheology, Vol. 38, No. 5-6, 2001, pp. 415-428.

[9] V. P. Srivastava, “A Theoretical Model for Blood Flow in Small Vessels,” International Journal of Application and Applied Mathematics, Vol. 2, No. 1, 2007, pp. 51-65.

[10] D. S. Sankar and U. Lee, "Two-Fluid Herchel-Bilkey Model for Blood Flow in Catheterized Arteries,” Journal of Mechanical Sciences and Technology, Vol. 22, No. 5, 2008, pp. 1008-1018. doi:10.1007/s12206-008-0123- 4

[11] B. K. Mishra and N. Verma, "Magnetic Effect on Blood Flow in a Multi-Stenosised Artery," Applied Mathematics and Computation, 2007.

[12] I. H. Chen, "Analysis of an Intensive Magnetic Field on Blood Flow: Part-2,” Journal of Bioelectronics, Vol. 4, No. 1, 1985, pp. 55-61.

[13] Y. Haik, V. Pai and C. J. Chen, "Apparent Viscosity of Human Blood in a High Statics Magnetic Field,” Journal of Magnetism and Magnetic Materials, Vol. 225, No. 1-2, 2001, pp. 180-186. doi:10.1016/S0304-8853(00)01249-X

[14] J. R. Womersley, "Method for the Calculation of Velocity Rate of Flow and Viscous Dragin Arteries When the Pressure Gradient is Known,” The Journal of Physiology, Vol. 127, No. 3, 1955, pp. 533-563. 
[15] E. N. Lightfoot, “Transport Phenomenon in Living System,” Wiley, New York, 1974.

[16] V. K. Sud and G. S. Sekhon, "Arterial Flow under Periodic Body Acceleration," Bulletin of Mathematical Biology, Vol. 47, No. 1, 1985, pp. 35-52.

[17] P. Chaturani and V. Palanisamy, "Pulsatile Flow of Bblood with Periodic Body Acceleration,” International Journal of Engineering Science, Vol. 29, No. 1, 1991, pp. 113-119. doi:10.1016/0020-7225(91)90081-D

[18] D. K. Wagh and S. D. Wagh, "Blood Flow Considered as Magnetic Flow," Proceeding of Physiology of fluid Dynamics III, 1992, pp. 311-315.

[19] P. G. Saffman, "On the Stability of Laminar Flow of a Dusty Gas,” The Journal of Fluid Mechanics, Vol. 13,
1962, pp. 120-128. doi:10.1017/S0022112062000555

[20] A. H. Nayfeh, "Osicillatory Two-Phase Flow Through a Rigid Pipe,” AIAA Journal, Vol. 4, 1966, pp. 1868-1871. doi:10.2514/3.3804

[21] D. C. Sanyal, K. Das and S. Debnath, "Effect of Magnetic Field on Pulsatile Blood Flow Through an Inclined Circular Tube with Periodic Body Acceleration,” The Journal of Physiological Sciences, Vol. 11, 2007, pp. 4356.

[22] T. Yamamoto, Y. Nagayama and M. Tamura, “A BloodOxygenation Dependent Increase in Blood Viscosity Due to a Static Magnetic Field," Physics in Medicine and Biology, Vol. 49, No. 14, 2004, pp. 3267-3277. doi:10.1088/0031-9155/49/14/017 